\title{
PERMANENCE STUDIES OF CURRENT COMMERCIAL BOOK PAPERS
}

\author{
By John O. Burton
}

\section{ABSTRACT}

This is one of a series of articles dealing with permanence studies of current commercial writing and printing papers. A wide variety of current book papers was tested for purity and strength and the effects of an artificial aging treatment on these papers were studied. In general, these book papers do not have as high purity as has been found in writing papers made of the same kind and quality of fibrous materials, nor do they resist to as great an extent the effects of the heat test used as an artificial aging treatment. The lower permanence qualities of the book papers are probably caused by the emphasis placed on printing quality instead of permanence qualities during their manufacture. The content of acid materials of many of the papers is quite high. Data previously procured indicate that the acidity of a permanent paper should not exceed the equivalent of 0.1 per cent sulphuric anhydride. This is further substantiated by the data obtained in the present study. There is a good correlation between the acidity of the paper and the change in strength and purity caused by the accelerated aging treatment.

Using the test results obtained as a basis, the papers were classified as to their relative permanence qualities. According to this classification system only one of the 28 papers studied could qualify as a permanent paper, but several were only slightly deficient. There was no constant relation between the quality of the raw materials used and the permanence qualities of the papers as judged from the tests applied.

\section{CONTENTS}

1. Introduction

II. Description of papers studied 430

III. Description of test methods employed... 431

1. Preparation of papers for testing

2. Physical tests

3. Chemical purity tests. 432

4. Accelerated aging test... 432

IV. Discussion of test data.

1. Strength tests

2. Permanence tests

(a) Chemical purity tests... 433

(b) Accelerated aging test._.................. 436

V. Classification of the papers relative to their permanence qualities._- 437

VI. Summary _... 439

VII. Conclusion

\section{INTRODUCTION}

The National Bureau of Standards, in cooperation with various interested governmental and private agencies, is studying problems connected with the permanence of papers used for records. A comprehensive program ${ }^{1}$ was formulated, part of which involved the testing of commercial writing and book papers and the fibrous raw

1 Scribner, B. W., Bureau of Standards Studies on the Deterioration of Paper, Pacific Pulp and Paper Industry, 4, No. 11, pp. 30-34; October, 1930. 
materials used in their manufacture to determine as far as possible their probable permanence. A report of the results obtained in the study of the paper-making fibers and of a wide range of current commercial writing papers has been published.2 A study of the permanence of representative commercial bond and ledger papers furnished by the United States Government Printing Office has been completed, and the results will be reported later. The present article contains the results of a study of current commercial book papers. The American Library Association and the National Association of Book Publishers both expressed their desire for this particular information. Thus, this article completes a rather thorough survey from a permanence standpoint of papers used for written and printed records that are obtainable on the market at the present time.

\section{DESCRIPTION OF PAPERS STUDIED}

An outline of the work planned was sent to several manufacturers of book papers with the request that they submit any papers which they considered suitable for the purpose. Some papers were also obtained from deliveries to the United States Government Printing Office. The papers received from these two sources are believed to be representative of current commercial book papers. Following is a list of the papers studied with the descriptions of the materials used in their preparation as furnished by the manufacturers. The numbers of the papers are those used throughout the article:

1. New cotton rags, 85 per cent, and new linen rags, 15 per cent; rosin sized.

2. Chemical wood fiber containing some highly purified wood fiber; rosin sized; clay filled.

3. Bleached cotton fiber, 75 per cent, and bleached sulphite pulp, 25 per cent; sized with 2 per cent rosin and alum.

4. Bleached sulphite pulp, 50 per cent, and unbleached sulphite pulp, 50 per cent; rosin sized; clay filled.

5. The same as paper No. 2, except that the highly purified wood fiber was replaced with an equal amount of cotton linters.

6. Rag fiber, 50 per cent, and sulphite pulp, 50 per cent; rosin sized; clay filled.

7. Rag fiber, 85 per cent, and sulphite pulp, 15 per cent; rosin sized; clay filled.

8. White, uncooked rags, 100 per cent; rosin sized.

9. Rag fiber, "twos and blues" grade, 55 per cent, and chemical wood fiber, 45 per cent; rosin sized; clay filled.

10. Rag fiber, "twos and blues" grade, 50 per cent, and chemical wood fiber, 50 per cent; rosin sized; clay filled.

11. Bleached chemical wood fiber, 100 per cent; rosin sized, clay filled.

12. Rag fiber, "twos and blues" grade, 100 per cent; rosin sized; clay filled.

13. Rag fiber, "No. 1 white" grade, 100 per cent; rosin sized; clay filled.

14. Rag fiber, "No. 2 white" grade, 100 per cent; rosin sized.

15. Rag fiber, "No. 2 white" grade, 100 per cent; rosin sized.

2 Rasch, R. H., A Study of Purified Wood Fibers as a Paper-Making Material, B. S. Jour. Research, 3, pp. 469-506; September, 1929. 
17. White linen and cotton rags, 100 per cent; rosin sized.

22. Rag fiber, 50 per cent, and bleached chemical wood fiber, 50 per cent; rosin sized; clay filled.

24. Bleached chemical wood fiber, 100 per cent; rosin sized; clay filled.

25. Rag fiber, 100 per cent; rosin sized; used for permanent editions of newspapers and periodicals.

28. Rag fiber, 50 per cent, and bleached chemical wood fiber, 50 per cent; rosin sized; clay filled.

31. Bleached chemical wood fiber, 100 per cent; double coated book paper.

32. Rag fiber, 50 per cent, and bleached chemical wood fiber, 50 per cent; double coated book paper.

37. New rags, 100 per cent; rosin sized.

38. "No. 1 white" grade of rags, 37.5 per cent, "thirds and blues" grade of rags, 37.5 per cent, and sulphite wood fiber', 25 per cent; rosin sized; clay filled.

40. Rag fiber, 50 per cent, bleached chemical wood fiber, 50 per cent; rosin sized; clay filled.

41. Rag fiber, 50per cent, and bleached chemical wood fiber, 50 per cent; double coated book paper.

45. New white and cream rags, 100 per cent; rosin sized. This paper was manufactured especially with a view to permanence.

46. Same as paper No. 45 but from a different delivery.

\section{DESCRIPTION OF TEST METHODS EMPLOYED}

\section{PREPARATION OF PAPERS FOR TESTING}

The samples for physical testing were conditioned and tested in a room maintained at a relative humidity of 65 per cent, \pm 2 per cent, and at a temperature of $21^{\circ}$ C., $\pm 2^{\circ}$ C. $\left(70^{\circ} \mathrm{F} . \pm 5^{\circ} \mathrm{F}.\right)$. These conditions are those specified by the paper testing committee of the technical Association of the Pulp and Paper Industry. The samples for the chemical purity tests were reduced to a uniform fibrous condition by means of mechanical disintegration. This method of preparation has been found necessary in order to insure complete reaction of the reagents with the fibers. A description of the grinder used and the reasons for this method of preparation have been given in full in previous publications. $^{3}$

\section{PHYSICAL TESTS}

The determinations of thickness, weight, fiber composition, opacity, bursting strength, tearing strength, and tensile strength were made by the official testing methods of the Technical Association of the Pulp and Paper Industry. ${ }^{4}$ The folding endurance was determined with a M. I. T. (Massachusetts Institute of Technology) type of tester, which is also described in Paper Testing Methods. This type of tester was used instead of the Schopper tester because it was necessary to use different tensions when testing different papers. Most of the book papers had insufficient strength to give a significant folding

\footnotetext{
3 Burton, J. O., and Rasch, R. H., The Determination of the Alpha Cellulose Content and Copper Number of Paper, B. S. Jour. Research, 6, pp. 603-619; April, 1931. See also footnote 2, p. 430.

1 Paper Testing Methods, prepared by the Committee on Paper Testing of the Technical Association of the Pulp and Paper Industry, Lockwood Trade J. Co., New York; 1929.
} 
endurance value when the Schopper machine available was used with the specified tension of $1 \mathrm{~kg}$. A folding endurance of at least 100 double folds was desired on all of the papers. To obtain this value the tension on the folding strip would have to be less than $1 \mathrm{~kg}$ for most of the papers. On the other hand, for accuracy and to save time in testing, the maximum tension consistent with obtaining a significant folding endurance value should be used. Therefore, different tensions were required for testing different papers. The tension on the paper strip in the Schopper tester may be varied only to a slight extent without changing the spring, and each change of tension requires a painstaking recalibration, whereas the tension on the strip in the M. I. T. tester may be varied over a much wider range and recalibrated with ease. Therefore, the latter type of tester was used. However, even with this machine it was not always possible to obtain a folding endurance value of 100 .

\section{CHEMICAL PURITY TESTS}

All of the chemical tests used have been described in the literature. The determinations of the amounts of rosin, ash, and casein were made by the official methods of the Technical Association of the Pulp and Paper Industry. ${ }^{5}$ The alphacellulose method ${ }^{6}$ was a modification of that of Jentgen, the copper-number method ${ }^{6}$ was essentially that of Braidy, and the acidity method ${ }^{7}$ was the one designed by Kohler and Hall.

\section{ACCELERATED AGING TEST}

A heat treatment at $100^{\circ} \mathrm{C} .\left(212^{\circ} \mathrm{F}\right.$.), for 72 hours was used to produce accelerated aging. The oven used for this purpose was equipped with a fan and duct for rapidly circulating the air. The temperature was controlled by means of a thermostat and relays to within $\pm 1^{\circ} \mathrm{C}$. $\left( \pm 2^{\circ}\right.$ F.). No attempt was made to purify the air nor to control its humidity. A detailed description and photograph of this oven were included in a previous publication. ${ }^{8}$ The effects of this treatment were measured by the change in the strength of the papers and changes in their chemical purity.

\section{DISCUSSION OF TEST DATA} them.

The test data are given in Table 1. Following is a discussion of

\section{STRENGTH TESTS}

It is difficult to compare the papers with one another as they vary widely in weight and finish. However, the strength of book papers is not such an important indication of quality as it is in the case of writing papers, since the latter are subjected to greater stresses. The folding endurance values obtained mean little in so far as the strengths of the papers are concerned, although they are significant in interpreting the effect of the aging treatment. This is because there is no common basis upon which the papers may be compared, since the

${ }^{5}$ See footnote 4.

- See footnote 3.

7. Kohler, and G. Hall, Acidity in Paper, The Paper Ind., 7, No. 7; October, 1925.

8 See footnute 2 , p. 430. 
tests were made at various tensions, and as far as is known there is no constant relation between the amount of tension and the folding value. The tearing strength and tensile strength are probably more indicative of the relative strengths of the papers. Some of the book papers compare very favorably with bond papers of the same weight in these two respects.

\section{PERMANENCE TESTS}

(a) CHEMICAL PURITY TESTS

The basic component of paper is cellulose, but several additional ingredients are added in the manufacturing process. For a permanent paper the cellulose composing the paper should have a high

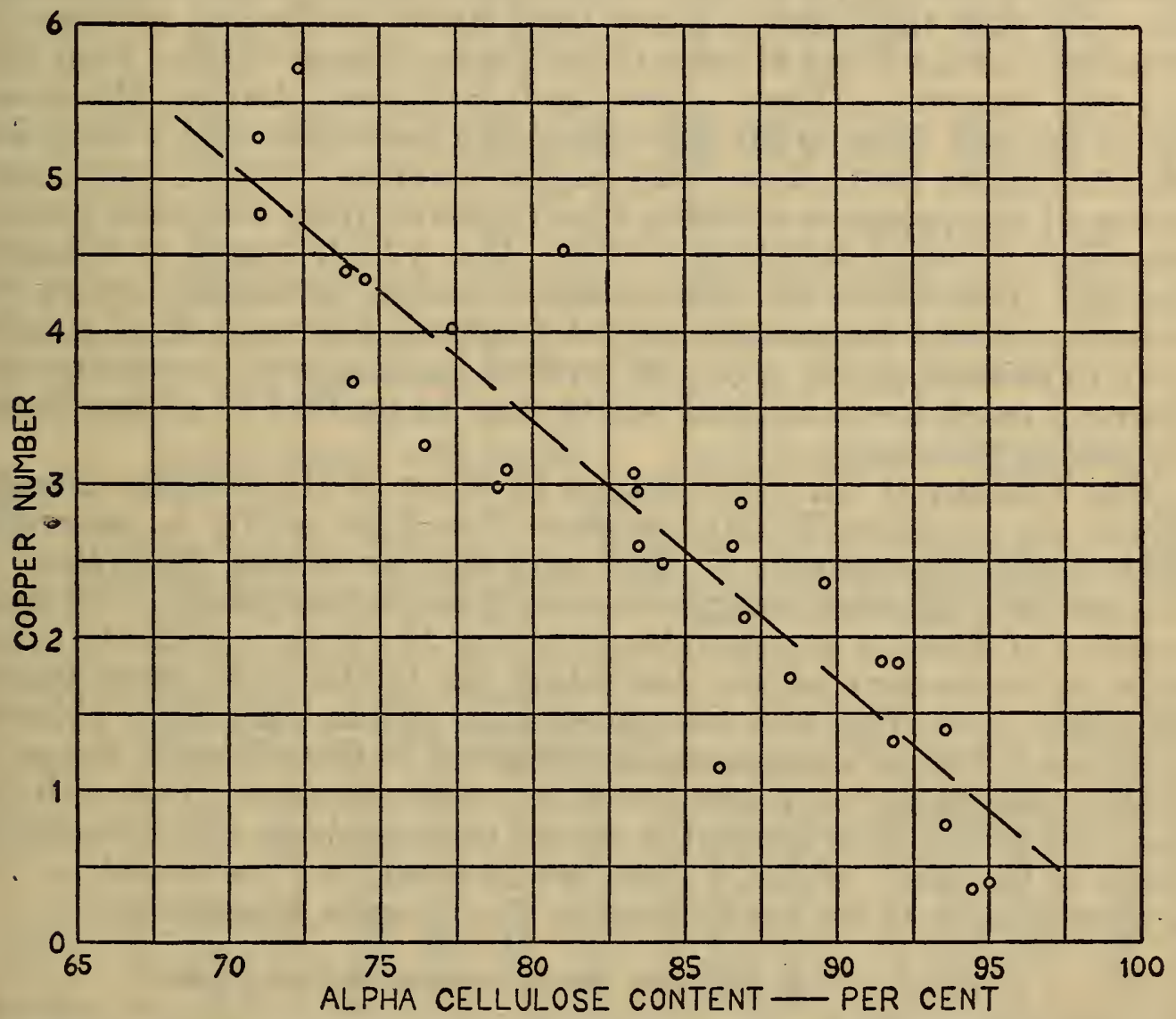

FIGURE 1.-Relation between the copper number and the alpha-cellulose content of the book papers studied

purity, and the amount of added ingredients, especially of those known to be harmful, should be a minimum. The copper number and the alpha-cellulose content are usually regarded as a measure of the purity of the cellulose. Papers of good quality are characterized by low copper numbers and high alpha-cellulose contents. Although there is no infallible rule relating these two purity tests, materials having a low copper number usually have a correspondingly high alpha-cellulose content. That this relation holds in general may be seen from Figure 1, where the copper numbers and alpha-cellulose contents of all the book papers studied are plotted with copper numbers as ordinates and alpha-cellulose contents as abscissas. There are nearly always two, and sometimes three, materials added $68723-31-2$ 
to book papers during the manufacturing process. These are rosin and alum for sizing and mineral filler for increasing the opacity and improving the printing quality. An excess of either of the first two is considered harmful. Excessive use of alum results in high acidity, which has a pronounced deteriorative effect upon paper. Nearly all mineral fillers used are chemically inert and are usually regarded as having no harmful effect upon the permanence of the paper.

The values obtained for the alpha-cellulose contents and the copper numbers (see Table 1 ) indicate a wide variation of purity among the different papers. In general, these book papers have a lower degree of purity than is found in writing papers made of the same quality of fibrous materials. This is probably due to the more severe cooking treatment given the fibers during book paper manufacture in order that the finished paper shall have the desired softness for printing; for the fibers of some of the papers appear to have been very severely treated. These papers, including many having fibers said to be derived from originally high-grade materials, had a very low alpha-cellulose content and high copper number. On the other hand, many of the papers containing fibers derived from materials usually regarded as being of inferior quality had a high degree of cellulosic purity. This shows the importance of careful treatment during the manufacture of permanent record papers. Fibers of high quality may have their purity seriously lowered by improper processing, and fibers of much lower original purity may be purified to a high degree by proper treatment.

The amount of titratable acidity in many of the samples is large. There are considerable data to show that high acidity is associated with rapid deterioration of paper and that an acidity equivalent to 0.1 per cent sulphuric anhydride should not be exceeded. The rosin content of most of the samples is within the limit considered desirable for permanent papers, indicating the tendency of paper manufacturers to use the least rosin possible to obtain the correct printing qualities. This is a commendable practice as there is good reason to believe that rosin has a harmful effect upon cellulose. But with the lowering of the rosin content it should be possible to avoid the use of much of the alum, which, if done, would result in a lower acidity. It is questionable if the use of rosin in book papers is necessary.

TABLE 1.-Test data on current commercial book papers

\begin{tabular}{|c|c|c|c|c|c|c|c|c|c|c|c|}
\hline \multirow[b]{2}{*}{ Sample No. } & \multicolumn{3}{|c|}{ Fiber composition } & \multicolumn{2}{|c|}{ Sizing } & \multirow[b]{2}{*}{ Ash } & \multirow{2}{*}{$\begin{array}{c}\text { Weight, } \\
500 \\
\text { sheets } \\
24 \text { by } 40 \\
\text { inches }\end{array}$} & \multirow[b]{2}{*}{$\begin{array}{c}\text { Thick- } \\
\text { ness }\end{array}$} & \multirow[b]{2}{*}{ Opacity } & \multicolumn{2}{|c|}{$\begin{array}{l}\text { Bursting } \\
\text { strength }\end{array}$} \\
\hline & Rag & $\begin{array}{l}\text { Chemi- } \\
\text { cal } \\
\text { wood, } \\
\text { conif- } \\
\text { erous }\end{array}$ & $\begin{array}{l}\text { Chemi- } \\
\text { cal } \\
\text { wood, } \\
\text { decid- } \\
\text { uous }\end{array}$ & Rosin & Casein & & & & & $\begin{array}{c}\text { Before } \\
\text { heat- } \\
\text { ing }\end{array}$ & $\begin{array}{l}\text { After } \\
\text { heat- } \\
\text { ing }\end{array}$ \\
\hline & \begin{tabular}{r} 
Per cent \\
100 \\
\hdashline 75 \\
\end{tabular} & $\begin{array}{r}\text { Pcr cent } \\
50 \\
15 \\
100\end{array}$ & $\begin{array}{r}\text { Per cent } \\
50 \\
10\end{array}$ & $\begin{array}{r}\text { Per cent } \\
1.2 \\
0.5 \\
1.6 \\
1.4 \\
0.7\end{array}$ & Per cent & $\begin{array}{r}\text { Percent } \\
0.60 \\
14.67 \\
1.61 \\
16.43 \\
15.53\end{array}$ & $\begin{array}{r}\text { Pounds } \\
53.9 \\
54.1 \\
38.5 \\
35.9 \\
54.0\end{array}$ & $\begin{array}{l}\text { Inch } \\
0.0037 \\
.0040 \\
.0030 \\
.0021 \\
.0041\end{array}$ & \begin{tabular}{|r|} 
Per cent \\
83.0 \\
92.9 \\
79.4 \\
86.1 \\
93.0
\end{tabular} & $\begin{array}{r}\text { Points } \\
34.6 \\
14.9 \\
15.2 \\
8.8 \\
11.7\end{array}$ & $\begin{array}{r}\text { Points } \\
30.0 \\
14.5 \\
14.0 \\
7.8 \\
11.2\end{array}$ \\
\hline & $\begin{array}{r}50 \\
90 \\
100 \\
30 \\
50\end{array}$ & $\begin{array}{l}10 \\
10\end{array}$ & & $\begin{array}{l}1.1 \\
1.0 \\
1.3 \\
1.7 \\
0.7\end{array}$ & & $\begin{array}{r}14.26 \\
12.35 \\
.18 \\
7.55 \\
14.43\end{array}$ & $\begin{array}{l}62.3 \\
62.2 \\
63.4 \\
40.5 \\
52.1\end{array}$ & $\begin{array}{l}.0052 \\
.0066 \\
.0073 \\
.0030 \\
.0030\end{array}$ & $\begin{array}{l}93.4 \\
94.0 \\
93.0 \\
85.0 \\
88.6\end{array}$ & $\begin{array}{l}19.3 \\
11.8 \\
13.8 \\
11.9 \\
11.7\end{array}$ & $\begin{array}{l}19.0 \\
12.1 \\
14.5 \\
10.7 \\
10.8\end{array}$ \\
\hline
\end{tabular}


TABLE 1.-Test data on current commercial book papers-Continued

\begin{tabular}{|c|c|c|c|c|c|c|c|c|c|c|c|}
\hline \multirow[b]{2}{*}{ Sample No. } & \multicolumn{3}{|c|}{ Fiber composition } & \multicolumn{2}{|c|}{ Sizing } & \multirow[b]{2}{*}{ Ash } & \multirow{2}{*}{$\mid \begin{array}{c}\text { Weight, } \\
500 \\
\text { sheets } \\
24 \text { by } 40 \\
\text { inches }\end{array}$} & \multirow[b]{2}{*}{$\begin{array}{c}\text { Thick- } \\
\text { ness }\end{array}$} & \multirow[b]{2}{*}{ Opacity } & \multicolumn{2}{|c|}{$\begin{array}{l}\text { Bursting } \\
\text { strength }\end{array}$} \\
\hline & Rag & $\begin{array}{c}\text { Chemi- } \\
\text { cal } \\
\text { wood, } \\
\text { conif- } \\
\text { erous }\end{array} \mid$ & $\begin{array}{c}\text { Chemi- } \\
\text { cal } \\
\text { wood, } \\
\text { decid- } \\
\text { uous }\end{array}$ & Rosin & Casein & & & & & $\begin{array}{c}\text { Before } \\
\text { heat- } \\
\text { ing }\end{array}$ & $\begin{array}{l}\text { After } \\
\text { heat- } \\
\text { ing }\end{array}$ \\
\hline $\begin{array}{l}11 . \\
12 . \\
13 . \\
14 . \\
15 .\end{array}$ & $\begin{array}{r}\text { Per cent } \\
100 \\
100 \\
100 \\
95\end{array}$ & $\begin{array}{r}P e r \text { cent } \\
40 \\
-\end{array}$ & $\begin{array}{r}\text { Per cent } \\
60\end{array}$ & $\begin{array}{r}\text { Per cent } \\
1.1 \\
1.3 \\
1.7 \\
2.4 \\
1.7\end{array}$ & $\begin{array}{l}\text { Per cent } \\
\\
\end{array}$ & $\begin{array}{r}\text { Per cent } \\
8.52 \\
7.97 \\
11.12 \\
3.91 \\
6.06\end{array}$ & $\begin{array}{r}\text { Pounds } \\
41.1 \\
53.1 \\
66.0 \\
41.8 \\
57.3\end{array}$ & $\begin{array}{l}\text { Inch } \\
0.0031 \\
.0038 \\
.0066 \\
.0041 \\
.0032\end{array}$ & \begin{tabular}{|r|} 
Per cent \\
84.4 \\
85.2 \\
93.4 \\
84.8 \\
90.0
\end{tabular} & $\begin{array}{r}\text { Points } \\
14.6 \\
17.8 \\
11.0 \\
10.0 \\
15.3\end{array}$ & $\begin{array}{r}\text { Points } \\
10.4 \\
16.3 \\
9.5 \\
9.5 \\
13.8\end{array}$ \\
\hline 24. & $\begin{array}{r}100 \\
50 \\
-100 \\
50\end{array}$ & $\begin{array}{r}30 \\
40 \\
50\end{array}$ & $\begin{array}{l}20 \\
60\end{array}$ & $\begin{array}{l}1.4 \\
2.5 \\
0.9 \\
1.0 \\
0.8\end{array}$ & & $\begin{array}{r}1.33 \\
5.50 \\
9.68 \\
.77 \\
11.00\end{array}$ & $\begin{array}{l}88.8 \\
43.5 \\
46.5 \\
40.7 \\
46.7\end{array}$ & $\begin{array}{l}.0118 \\
.0035 \\
.0026 \\
.0030 \\
.0030\end{array}$ & \begin{tabular}{l|}
94.9 \\
87.1 \\
83.1 \\
81.5 \\
85.6
\end{tabular} & \begin{tabular}{l|}
33.6 \\
13.1 \\
13.0 \\
14.6 \\
14.4
\end{tabular} & $\begin{array}{r}33.3 \\
9.5 \\
10.8 \\
14.8 \\
13.9\end{array}$ \\
\hline $\begin{array}{l}31 . \\
32- \\
37- \\
38 .\end{array}$ & $\begin{array}{r}60 \\
100 \\
75\end{array}$ & $\begin{array}{r}70 \\
40 \\
-25 \\
25\end{array}$ & 30 & \begin{tabular}{l|}
1.0 \\
1.4 \\
1.2 \\
1.4
\end{tabular} & $\begin{array}{l}4.90 \\
5.96\end{array}$ & $\begin{array}{r}24.00 \\
23.30 \\
.64 \\
12.68\end{array}$ & $\begin{array}{l}70.2 \\
87.0 \\
45.1 \\
41.9\end{array}$ & $\begin{array}{l}.0034 \\
.0042 \\
.0071 \\
.0027\end{array}$ & \begin{tabular}{l|}
95.0 \\
97.7 \\
96.2 \\
86.7
\end{tabular} & \begin{tabular}{l|}
20.4 \\
31.8 \\
25.7 \\
13.6
\end{tabular} & $\begin{array}{l}18.7 \\
30.8 \\
26.2 \\
12.5\end{array}$ \\
\hline $\begin{array}{l}40 .- \\
41-- \\
45 .- \\
46 .-\end{array}$ & $\begin{array}{r}50 \\
60 \\
100 \\
100\end{array}$ & $\begin{array}{l}50 \\
35\end{array}$ & & $\begin{array}{l}2.0 \\
1.5 \\
1.7\end{array}$ & $5 . \overline{8}$ & $\begin{array}{r}9.87 \\
24.86 \\
.29 \\
.20\end{array}$ & $\begin{array}{l}40.0 \\
89.3 \\
77.7 \\
76.7\end{array}$ & $\begin{array}{l}.0031 \\
.0042 \\
.0063 \\
.0050\end{array}$ & \begin{tabular}{c}
86.6 \\
97.0 \\
\hdashline.--
\end{tabular} & $\begin{array}{l}10.8 \\
28.4 \\
44.9 \\
52.3\end{array}$ & $\begin{array}{l}12.3 \\
27.9 \\
44.1 \\
51.5\end{array}$ \\
\hline
\end{tabular}

\begin{tabular}{|c|c|c|c|c|c|c|c|c|c|c|c|c|c|c|c|}
\hline \multirow{3}{*}{$\begin{array}{l}\text { Sample } \\
\text { No. }\end{array}$} & \multicolumn{8}{|c|}{ Folding endurance } & \multicolumn{7}{|c|}{ Tearing strength } \\
\hline & \multirow[b]{2}{*}{$\begin{array}{l}\text { Ten- } \\
\text { sion }\end{array}$} & \multicolumn{3}{|c|}{ Machine direction } & \multicolumn{3}{|c|}{$\begin{array}{l}\text { Across machine } \\
\text { direction }\end{array}$} & \multirow{2}{*}{$\begin{array}{l}\text { Aver- } \\
\text { age } \\
\text { reten- } \\
\text { tion }\end{array}$} & \multicolumn{3}{|c|}{ Machine direction } & \multicolumn{3}{|c|}{$\begin{array}{l}\text { Across machine } \\
\text { direction }\end{array}$} & \multirow{2}{*}{$\begin{array}{c}\text { Aver } \\
\text { age } \\
\text { reten } \\
\text { tion }\end{array}$} \\
\hline & & $\begin{array}{c}\text { Be- } \\
\text { fore } \\
\text { heat- } \\
\text { ing }\end{array}$ & $\begin{array}{l}\text { After } \\
\text { heat- } \\
\text { ing }\end{array}$ & $\begin{array}{l}\text { Re- } \\
\text { ten- } \\
\text { tion }\end{array}$ & $\begin{array}{c}\text { Be- } \\
\text { fore } \\
\text { heat- } \\
\text { ing }\end{array}$ & $\begin{array}{c}\text { After } \\
\text { heat- } \\
\text { ing }\end{array}$ & $\begin{array}{c}\text { Re- } \\
\text { ten- } \\
\text { tion }\end{array}$ & & $\begin{array}{c}\text { Be- } \\
\text { fore } \\
\text { heat- } \\
\text { ing }\end{array}$ & $\begin{array}{c}\text { After } \\
\text { heat- } \\
\text { ing }\end{array}$ & $\begin{array}{c}\text { Re- } \\
\text { ten- } \\
\text { tion }\end{array}$ & $\begin{array}{c}\text { Be- } \\
\text { fore } \\
\text { heat- } \\
\text { ing }\end{array}$ & $\begin{array}{c}\text { After } \\
\text { heat- } \\
\text { ing }\end{array}$ & $\begin{array}{c}\text { Re- } \\
\text { ten- } \\
\text { tion }\end{array}$ & \\
\hline & $\begin{array}{r}\mathrm{kg} \\
1.0 \\
.5 \\
.5 \\
.4 \\
.4\end{array}$ & $\begin{array}{c}\text { Dou } \\
\text { ble } \\
\text { folds } \\
159 \\
36 \\
142 \\
18 \\
23\end{array}$ & $\begin{array}{c}\text { Dou- } \\
\text { ble } \\
\text { folds } \\
75 \\
29 \\
81 \\
11 \\
16\end{array}$ & $\begin{array}{r}\text { Per } \\
\text { cent } \\
47 \\
81 \\
57 \\
61 \\
70\end{array}$ & $\begin{array}{c}\text { Dou- } \\
\text { ble } \\
\text { folds } \\
98 \\
27 \\
39 \\
18 \\
18\end{array}$ & 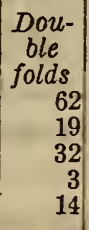 & \begin{tabular}{r|} 
Per \\
cent \\
63 \\
70 \\
82 \\
17 \\
78
\end{tabular} & \begin{tabular}{|r} 
Per \\
cent \\
55 \\
76 \\
70 \\
39 \\
$\mathbf{7 4}$
\end{tabular} & \begin{tabular}{|r|}
$g$ \\
100.4 \\
57.0 \\
42.8 \\
25.3 \\
37.6
\end{tabular} & $\begin{array}{l}g \\
66.4 \\
55.0 \\
43.6 \\
16.8 \\
36.4\end{array}$ & $\begin{array}{r}\text { Per } \\
\text { cent } \\
66 \\
96 \\
102 \\
66 \\
97\end{array}$ & \begin{tabular}{r}
\multicolumn{1}{c}{$g$} \\
104.0 \\
42.6 \\
37.8 \\
24.3 \\
36.8
\end{tabular} & \begin{tabular}{l|}
$g$ \\
68.4 \\
49.2 \\
33.4 \\
14.7 \\
32.6
\end{tabular} & \begin{tabular}{|r|} 
Per \\
cent \\
66 \\
115 \\
88 \\
60 \\
89
\end{tabular} & $\begin{array}{r}\text { Per } \\
\text { cent } \\
66 \\
106 \\
95 \\
63 \\
93\end{array}$ \\
\hline & $\begin{array}{l}.5 \\
.3 \\
.3 \\
.5 \\
.5\end{array}$ & $\begin{array}{r}299 \\
57 \\
119 \\
16 \\
12\end{array}$ & $\begin{array}{r}174 \\
41 \\
88 \\
10 \\
5\end{array}$ & $\begin{array}{l}58 \\
72 \\
74 \\
63 \\
42\end{array}$ & $\begin{array}{l}68 \\
27 \\
50 \\
16 \\
11\end{array}$ & $\begin{array}{r}52 \\
21 \\
37 \\
10 \\
5\end{array}$ & $\begin{array}{l}76 \\
78 \\
74 \\
63 \\
45\end{array}$ & $\begin{array}{l}67 \\
75 \\
74 \\
63 \\
44\end{array}$ & $\begin{array}{l}78.4 \\
46.4 \\
58.6 \\
33.2 \\
35.2\end{array}$ & $\begin{array}{l}70.0 \\
46.4 \\
53.4 \\
26.0 \\
28.4\end{array}$ & $\begin{array}{r}90 \\
100 \\
91 \\
78 \\
81\end{array}$ & $\begin{array}{l}76.4 \\
42.0 \\
50.4 \\
30.4 \\
31.2\end{array}$ & \begin{tabular}{l|}
67.6 \\
39.8 \\
50.6 \\
27.2 \\
27.2
\end{tabular} & $\begin{array}{r}88 \\
95 \\
100 \\
89 \\
87\end{array}$ & $\begin{array}{l}9 \\
9 \\
0\end{array}$ \\
\hline & $\begin{array}{l}.5 \\
.5 \\
.3 \\
.5 \\
.5\end{array}$ & $\begin{array}{l}53 \\
40 \\
21 \\
17 \\
41\end{array}$ & $\begin{array}{r}3 \\
13 \\
7 \\
7 \\
17\end{array}$ & $\begin{array}{r}6 \\
33 \\
33 \\
41 \\
41\end{array}$ & $\begin{array}{r}32 \\
48 \\
18 \\
9 \\
23\end{array}$ & $\begin{array}{r}4 \\
17 \\
7 \\
6 \\
15\end{array}$ & $\begin{array}{l}13 \\
35 \\
39 \\
67 \\
65\end{array}$ & $\begin{array}{l}10 \\
34 \\
36 \\
54 \\
53\end{array}$ & $\begin{array}{l}36.6 \\
43.2 \\
54.4 \\
38.2 \\
49.0\end{array}$ & $\begin{array}{l}18.4 \\
38.4 \\
45.0 \\
32.6 \\
37.6\end{array}$ & $\begin{array}{l}50 \\
89 \\
83 \\
85 \\
77\end{array}$ & $\begin{array}{l}29.2 \\
44.0 \\
47.4 \\
32.6 \\
38.9\end{array}$ & $\begin{array}{l}14.8 \\
34.0 \\
45.0 \\
31.0 \\
31.8\end{array}$ & $\begin{array}{l}50 \\
77 \\
95 \\
95 \\
82\end{array}$ & 8 \\
\hline & $\begin{array}{l}.5 \\
.5 \\
.5 \\
.5 \\
.5\end{array}$ & $\begin{array}{r}168 \\
118 \\
27 \\
299 \\
171\end{array}$ & $\begin{array}{r}56 \\
9 \\
10 \\
316 \\
77\end{array}$ & $\begin{array}{r}33 \\
8 \\
37 \\
106 \\
45\end{array}$ & $\begin{array}{r}135 \\
35 \\
25 \\
138 \\
66\end{array}$ & $\begin{array}{r}69 \\
8 \\
10 \\
100 \\
43\end{array}$ & $\begin{array}{l}51 \\
23 \\
40 \\
72 \\
65\end{array}$ & $\begin{array}{l}42 \\
16 \\
39 \\
89 \\
55\end{array}$ & $\begin{array}{l}81.2 \\
38.8 \\
36.8 \\
58.4 \\
45.0\end{array}$ & $\begin{array}{l}82.0 \\
35.2 \\
26.4 \\
62.4 \\
45.4\end{array}$ & $\begin{array}{r}101 \\
91 \\
72 \\
107 \\
101\end{array}$ & $\begin{array}{l}80.4 \\
31.0 \\
31.4 \\
60.0 \\
41.6\end{array}$ & $\begin{array}{l}79.6 \\
34.8 \\
23.0 \\
57.8 \\
38.0\end{array}$ & $\begin{array}{r}99 \\
112 \\
73 \\
96 \\
91\end{array}$ & $\begin{array}{r}100 \\
102 \\
73 \\
102 \\
96\end{array}$ \\
\hline & $\begin{array}{l}.5 \\
.8 \\
.5 \\
.5\end{array}$ & \begin{tabular}{r|}
21 \\
248 \\
177 \\
288
\end{tabular} & $\begin{array}{r}18 \\
162 \\
158 \\
187\end{array}$ & $\begin{array}{l}86 \\
65 \\
89 \\
65\end{array}$ & $\begin{array}{l}34 \\
82 \\
74 \\
54\end{array}$ & $\begin{array}{l}28 \\
67 \\
87 \\
53\end{array}$ & $\begin{array}{r}82 \\
82 \\
118 \\
98\end{array}$ & $\begin{array}{r}84 \\
74 \\
104 \\
82\end{array}$ & $\begin{array}{l}36.0 \\
76.4 \\
98.0 \\
61.8\end{array}$ & $\begin{array}{l}32.2 \\
71.4 \\
97.2 \\
55.6\end{array}$ & $\begin{array}{l}89 \\
93 \\
99 \\
90\end{array}$ & $\begin{array}{l}36.4 \\
69.6 \\
99.2 \\
56.8\end{array}$ & \begin{tabular}{l|}
30.6 \\
63.4 \\
99.2 \\
49.8
\end{tabular} & $\begin{array}{r}84 \\
91 \\
100 \\
88\end{array}$ & $\begin{array}{r}87 \\
92 \\
100 \\
89\end{array}$ \\
\hline 41. & (1) $^{.5} .7$ & $\begin{array}{r}54 \\
213 \\
740 \\
1,699\end{array}$ & $\begin{array}{r}30 \\
105 \\
702 \\
1,331\end{array}$ & $\begin{array}{l}56 \\
49 \\
95 \\
78\end{array}$ & $\begin{array}{r}12 \\
93 \\
289 \\
639\end{array}$ & $\begin{array}{r}12 \\
68 \\
203 \\
500\end{array}$ & $\begin{array}{r}100 \\
73 \\
70 \\
78\end{array}$ & $\begin{array}{l}78 \\
61 \\
83 \\
78\end{array}$ & $\begin{array}{r}38.0 \\
66.0 \\
136.8 \\
121.6\end{array}$ & $\begin{array}{r}37.2 \\
60.8 \\
124.8 \\
115.6\end{array}$ & $\begin{array}{l}98 \\
92 \\
91 \\
95\end{array}$ & $\begin{array}{r}33.6 \\
57.2 \\
140.8 \\
124.4\end{array}$ & \begin{tabular}{r|}
37.6 \\
31.6 \\
133.2 \\
122.0
\end{tabular} & $\begin{array}{r}112 \\
55 \\
95 \\
98\end{array}$ & $\begin{array}{r}105 \\
74 \\
93 \\
97\end{array}$ \\
\hline
\end{tabular}

1 The folding endurance of papers 45 and 46 was determined with the Schopper folding endurance machine 
TABLE 1.-Test data on current commercial book papers-Continued

\begin{tabular}{|c|c|c|c|c|c|c|c|c|c|c|c|c|c|c|c|}
\hline \multirow{3}{*}{$\begin{array}{c}\text { Sample } \\
\text { No. }\end{array}$} & \multicolumn{7}{|c|}{ Tensile strength } & \multicolumn{3}{|c|}{$\begin{array}{l}\text { Alpha-cellulose } \\
\text { content }\end{array}$} & \multicolumn{3}{|c|}{ Copper No. } & \multicolumn{2}{|c|}{ Acidity } \\
\hline & \multicolumn{3}{|c|}{$\begin{array}{c}\text { Machine direc- } \\
\text { tion }\end{array}$} & \multicolumn{3}{|c|}{$\begin{array}{l}\text { Across machine } \\
\text { direction }\end{array}$} & \multirow{2}{*}{$\begin{array}{c}\text { Aver- } \\
\text { age } \\
\text { re- } \\
\text { ten- } \\
\text { tion }\end{array}$} & \multirow{2}{*}{$\begin{array}{c}\text { Be- } \\
\text { fore } \\
\text { heat- } \\
\text { ing }\end{array}$} & \multirow{2}{*}{$\begin{array}{c}\text { After } \\
\text { heat- } \\
\text { ing }\end{array}$} & \multirow[b]{2}{*}{ Loss } & \multirow{2}{*}{$\begin{array}{c}\text { Be- } \\
\text { fore } \\
\text { heat- } \\
\text { ing }\end{array}$} & \multirow{2}{*}{$\begin{array}{c}\text { After } \\
\text { heat- } \\
\text { ing }\end{array}$} & \multirow{2}{*}{$\begin{array}{c}\text { In- } \\
\text { crease }\end{array}$} & \multirow{2}{*}{$\begin{array}{l}\text { Sul- } \\
\text { phuric } \\
\text { anhy- } \\
\text { dride }\end{array}$} & \multirow{2}{*}{$\begin{array}{l}\text { Acid, } \\
\text { num- } \\
\text { ber }\end{array}$} \\
\hline & $\begin{array}{c}\text { Be- } \\
\text { fore } \\
\text { heat- } \\
\text { ing }\end{array}$ & $\begin{array}{c}\text { After } \\
\text { heat- } \\
\text { ing }\end{array}$ & $\begin{array}{l}\text { Re- } \\
\text { ten- } \\
\text { tion }\end{array}$ & $\begin{array}{c}\text { Be- } \\
\text { fore } \\
\text { heat- } \\
\text { ing }\end{array}$ & $\begin{array}{c}\text { After } \\
\text { heat- } \\
\text { ing }\end{array}$ & $\begin{array}{c}\text { Re- } \\
\text { ten- } \\
\text { tion }\end{array}$ & & & & & & & & & \\
\hline & \begin{tabular}{l|}
$\mathrm{kg}$ \\
6.90 \\
3.92 \\
3.35 \\
2.26 \\
3.07
\end{tabular} & \begin{tabular}{|l|}
$k g$ \\
6.71 \\
3.89 \\
3.46 \\
2.16 \\
2.85
\end{tabular} & $\begin{array}{r}\text { Per } \\
\text { cent } \\
97 \\
99 \\
103 \\
96 \\
93\end{array}$ & $\begin{array}{l}\mathrm{kg} \\
4.18 \\
2.17 \\
1.91 \\
1.29 \\
1.76\end{array}$ & $\begin{array}{l}k g \\
3.95 \\
2.11 \\
1.77 \\
1.38 \\
1.70\end{array}$ & $\begin{array}{r}\text { Per } \\
\text { cent } \\
95 \\
97 \\
93 \\
107 \\
97\end{array}$ & $\begin{array}{r}\text { Per } \\
\text { cent } \\
96 \\
98 \\
98 \\
102 \\
95\end{array}$ & $\begin{array}{l}\text { Per } \\
\text { cent } \\
86.56 \\
76.53 \\
86.98 \\
81.04 \\
78.91\end{array}$ & $\begin{array}{l}\text { Per } \\
\text { cent } \\
82.36 \\
76.26 \\
82.80 \\
78.38 \\
77.55\end{array}$ & $\begin{array}{c}\text { Per } \\
\text { cent } \\
4.20 \\
.27 \\
4.18 \\
2.66 \\
1.36\end{array}$ & $\begin{array}{l}2.56 \\
3.25 \\
2.14 \\
4.53 \\
2.98\end{array}$ & $\begin{array}{l}3.09 \\
3.64 \\
2.73 \\
4.98 \\
3.22\end{array}$ & $\begin{array}{r}0.53 \\
.39 \\
.59 \\
.45 \\
.24\end{array}$ & \begin{tabular}{|l|} 
Per \\
cent \\
0.096 \\
.024 \\
.120 \\
.148 \\
.032
\end{tabular} & $\begin{array}{r}24 \\
6 \\
30 \\
37 \\
8\end{array}$ \\
\hline & $\begin{array}{l}\text { 5. } 60 \\
\text { 3. } 48 \\
\text { 3. } 93 \\
\text { 3. } 08 \\
\text { 3. } 16\end{array}$ & \begin{tabular}{l|}
5.51 \\
3.50 \\
4.10 \\
2.90 \\
2.93
\end{tabular} & $\begin{array}{r}98 \\
101 \\
104 \\
94 \\
93\end{array}$ & \begin{tabular}{r|}
2.31 \\
1.65 \\
2.04 \\
1.86 \\
1.78
\end{tabular} & $\begin{array}{l}2.30 \\
1.73 \\
2.18 \\
1.79 \\
1.72\end{array}$ & $\begin{array}{r}100 \\
105 \\
107 \\
96 \\
97\end{array}$ & $\begin{array}{r}99 \\
103 \\
106 \\
95 \\
95\end{array}$ & $\begin{array}{l}89.60 \\
91.98 \\
93.54 \\
74.09 \\
70.95\end{array}$ & $\begin{array}{l}84.70 \\
83.68 \\
85.99 \\
68.75 \\
63.93\end{array}$ & $\begin{array}{l}4.90 \\
8.30 \\
7.55 \\
5.34 \\
7.02\end{array}$ & \begin{tabular}{|}
2.34 \\
1.82 \\
1.41 \\
3.69 \\
5.33
\end{tabular} & $\begin{array}{l}2.80 \\
2.39 \\
1.90 \\
4.29 \\
5.70\end{array}$ & $\begin{array}{l}.52 \\
.57 \\
.49 \\
.60 \\
.37\end{array}$ & $\begin{array}{l}.048 \\
.020 \\
.040 \\
.232 \\
.188\end{array}$ & $\begin{array}{r}12 \\
5 \\
10 \\
58 \\
47\end{array}$ \\
\hline 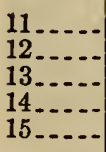 & $\begin{array}{l}3.78 \\
4.20 \\
3.19 \\
2.64 \\
4.02\end{array}$ & $\begin{array}{l}3.62 \\
3.86 \\
2.84 \\
2.51 \\
3.82\end{array}$ & $\begin{array}{l}96 \\
92 \\
89 \\
95 \\
95\end{array}$ & $\begin{array}{l}1.83 \\
2.56 \\
1.96 \\
1.26 \\
2.19\end{array}$ & $\begin{array}{l}1.61 \\
2.37 \\
1.83 \\
1.28 \\
2.08\end{array}$ & $\begin{array}{r}88 \\
93 \\
93 \\
102 \\
95\end{array}$ & $\begin{array}{l}92 \\
93 \\
91 \\
99 \\
95\end{array}$ & $\begin{array}{l}71.08 \\
73.90 \\
72.23 \\
83.49 \\
77.37\end{array}$ & $\begin{array}{l}58.61 \\
69.53 \\
66.87 \\
77.63 \\
71.16\end{array}$ & $\begin{array}{r}12.47 \\
4.37 \\
5.36 \\
5.86 \\
6.21\end{array}$ & $\mid \begin{array}{l}4.75 \\
4.40 \\
5.75 \\
2.61 \\
4.03\end{array}$ & $\begin{array}{l}5.56 \\
4.88 \\
6.15 \\
3.17 \\
4.32\end{array}$ & $\begin{array}{l}.80 \\
.48 \\
.40 \\
.56 \\
.29\end{array}$ & $\begin{array}{l}.240 \\
.180 \\
.152 \\
.184 \\
.208\end{array}$ & $\begin{array}{l}60 \\
45 \\
38 \\
46 \\
52\end{array}$ \\
\hline $\begin{array}{l}22 . . \\
24 \ldots \\
25 \ldots \\
28 \ldots\end{array}$ & $\begin{array}{l}6.93 \\
3.31 \\
3.70 \\
3.10 \\
4.00\end{array}$ & $\begin{array}{l}6.82 \\
2.69 \\
3.50 \\
3.20 \\
3.80\end{array}$ & $\begin{array}{r}98 \\
81 \\
95 \\
103 \\
95\end{array}$ & $\begin{array}{l}4.92 \\
1.56 \\
1.90 \\
1.80 \\
2.00\end{array}$ & $\begin{array}{l}5.05 \\
1.39 \\
1.90 \\
1.90 \\
2.00\end{array}$ & $\begin{array}{r}103 \\
89 \\
100 \\
106 \\
100\end{array}$ & $\begin{array}{r}101 \\
85 \\
98 \\
105 \\
98\end{array}$ & $\begin{array}{l}88.45 \\
83.31 \\
74.43 \\
91.92 \\
83.40\end{array}$ & \begin{tabular}{|l|}
84.02 \\
67.68 \\
68.42 \\
91.06 \\
81.37
\end{tabular} & $\begin{array}{r}4.43 \\
15.63 \\
6.01 \\
.86 \\
2.03\end{array}$ & $\begin{array}{l}1.72 \\
3.07 \\
4.35 \\
1.32 \\
2.95\end{array}$ & $\begin{array}{l}2.19 \\
4.17 \\
4.84 \\
1.46 \\
3.28\end{array}$ & $\begin{array}{r}.47 \\
1.10 \\
.49 \\
.14 \\
.33\end{array}$ & $\begin{array}{r}.152 \\
.196 \\
.196 \\
.080 \\
.092\end{array}$ & $\begin{array}{l}20 \\
23\end{array}$ \\
\hline 37. & $\begin{array}{l}5.15 \\
7.20 \\
6.10 \\
3.30\end{array}$ & $\begin{array}{l}4.82 \\
6.93 \\
6.30 \\
3.20\end{array}$ & $\begin{array}{r}94 \\
96 \\
103 \\
97\end{array}$ & $\begin{array}{l}\text { 3. } 14 \\
\text { 3. } 77 \\
3.70 \\
1.60\end{array}$ & $\begin{array}{l}2.85 \\
3.66 \\
3.70 \\
1.50\end{array}$ & $\begin{array}{r}91 \\
97 \\
100 \\
94\end{array}$ & $\begin{array}{r}93 \\
97 \\
102 \\
96\end{array}$ & $\begin{array}{l}79.23 \\
86.09 \\
93.36 \\
91.51\end{array}$ & $\begin{array}{l}76.56 \\
85.82 \\
92.16 \\
90.21\end{array}$ & $\begin{array}{l}2.67 \\
.27 \\
1.20 \\
1.30\end{array}$ & \begin{tabular}{r|}
3.10 \\
1.13 \\
.77 \\
1.85
\end{tabular} & $\begin{array}{l}3.18 \\
1.77 \\
1.02 \\
2.37\end{array}$ & $\begin{array}{l}.08 \\
.64 \\
.25 \\
.51\end{array}$ & $\begin{array}{r}.060 \\
.048\end{array}$ & 15 \\
\hline & $\begin{array}{r}3.50 \\
7.30 \\
9.27 \\
10.02\end{array}$ & $\begin{array}{l}3.70 \\
7.20 \\
9.22 \\
9.93\end{array}$ & $\begin{array}{r}106 \\
99 \\
99 \\
99\end{array}$ & $\begin{array}{l}1.60 \\
3.80 \\
5.30 \\
6.22\end{array}$ & $\begin{array}{l}1.70 \\
3.80 \\
5.47 \\
6.33\end{array}$ & $\begin{array}{l}106 \\
100 \\
103 \\
102\end{array}$ & $\begin{array}{l}106 \\
100 \\
101 \\
101\end{array}$ & $\begin{array}{l}86.92 \\
84.20 \\
95.00 \\
94.40\end{array}$ & $\begin{array}{l}85.23 \\
82.10 \\
92.49 \\
92.38\end{array}$ & $\begin{array}{l}1.69 \\
2.10 \\
2.51 \\
2.02\end{array}$ & $\begin{array}{r}2.87 \\
2.48 \\
.40 \\
.35\end{array}$ & $\begin{array}{r}3.34 \\
2.95 \\
.93 \\
.64\end{array}$ & $\begin{array}{l}.47 \\
.47 \\
.53 \\
.29\end{array}$ & $\begin{array}{c}.068 \\
.028 \\
.015\end{array}$ & $\begin{array}{r}17 \\
7 \\
4\end{array}$ \\
\hline
\end{tabular}

2 The acid number is the number of $\mathrm{ml}$ of $0.01 \mathrm{~N} \mathrm{NaOH}$ required to neutralize the three successive hot water extracts of $10 \mathrm{~g}$ of ground paper.

\section{(b) ACCELERATED AGING TEST}

The effect of the accelerated aging treatment, heating for 72 hours at $100^{\circ} \mathrm{C} .\left(212^{\circ} \mathrm{F}\right.$.), is shown by the change in strength of the papers and changes in their chemical purity. In an investigation such as this where a number of kinds of papers from a variety of sources is being studied there are too many variables to obtain very definite relationships among the properties determined. However, several general relationships may be observed.

A high content of acid materials is accompanied by a correspondingly large decrease in alpha-cellulose content. It is noticeable that all of the papers appear to fall into two groups. One of these groups contains papers which lose less than 2 per cent alpha cellulose during the heat treatment, and the other group contains papers which lose more than 4 per cent. The papers in the first group have an acidity less than 0.1 per cent sulphuric anhydride, and those in the second group have an acidity greater than 0.1 per cent sulphuric anhydride. Papers 6,7 , and 8 are exceptions to this classification. These three papers were made by the same manufacturer and according to his 
description were made from good materials. They had low acidities, but the decrease of alpha-cellulose content of all three was high. This large decrease in alpha-cellulose content during the heat treatment may have been caused by a too severe treatment of the stock during manufacture.

The increase of the copper number by the heat treatment was not particularly significant. There is a very general relationship in that the best papers, judged from the other tests, increased very little in copper number. Other than this there appeared to be no constant relation between increase in copper number and the other tests.

The loss of strength caused by the heat treatment is best measured by the change in folding endurance. The other strength factors are but slightly changed by this treatment and the more sensitive folding endurance test is the best criterion of loss in strength. There was a general relationship between the chemical purity tests and the retention of folding strength after the accelerated aging treatment. Papers which retained the most strength decreased the least in alpha-cellulose content. There was again the occurrence of two distinct groups of papers. Those which decreased less than 2 per cent in alpha-cellulose content retained more than 70 per cent of their folding strength. Those which decreased more than 4 per cent in alpha-cellulose content retained less than 70 per cent of their folding strength. Samples 6,7 , and 8 are again exceptions to this grouping.

\section{CLASSIFICATION OF THE PAPERS RELATIVE TO THEIR PERMANENCE QUALITIES}

A system of classification of printing and writing papers relative to their permanence qualities has been suggested by the National Bureau of Standards. ${ }^{9}$ This classification is based upon the purity and strength of the finished paper. The values given are derived from the results of the extensive investigations of this bureau during the past four years on the permanence of paper. In these investigations tests similar to the ones described here have been made on a large number and variety of papers, and it is believed that papers fulfilling the requirements of the various groups will have the relative degree of permanence indicated.

In this system of classification all papers are divided into four classes. The first class contains those papers which may be expected to have maximum longevity. A book paper fulfiling the requirements of this class would be particularly suitable for bound volumes of records having permanent value. The second class contains papers of high purity. Papers used for semipermanent records and in fine editions of rare and costly books should fulfill the requirements of this class. The third class is composed of papers which, while not having as high a degree of purity as those in the first two classes, have a fair degree of purity. Such papers could be used where moderate longevity is desired, such as in reference periodicals. Papers in the fourth class are those suitable for current use only. Table 2 illustrates in detail how such a system of classification might be applied to book papers. Of the book papers studied in the present investiga-

B. W. Scribner, Permanence Standards for Printing and Writing Papers, Trans. A. S. M. E., 52, No. 19, P. I. 52-55; May-August, 1930. Also, Paper Mill, 53, p. 13; June 21, 1930. 
tion No. 37 is the only one that fulfills completely the requirements of Class I. Some of the other papers fulfill the requirements of this class except in one or two respects. Nos. $25,32,38,45$, and 46 fall in Class II ; Nos. $1,2,3,5,6,7,8,17,28,31,40$, and 41, in Class III; and the remainder in Class IV.

\section{TABLE 2.-Classification of book papers}

\section{Class I. PAPERS OF MAXIMUM PURITY FOR PERMANENT RECORDS}

Stock: Free from unbleached or ground wood fibers. Alpha cellulose content not less than 90 per cent. Copper number not more than 1.0.

Acidity: $\mathrm{pH}$ value not less than 4.5 and acid number less than 25

Rosin: Not more than 1.0 per cent.

Stability: When heated for 72 hours at $100^{\circ} \mathrm{C}$. the alpha-cellulose content shall decrease not more than 1.5 per cent, the folding endurance shall decrease not more than 25 per cent, and the copper number shall increase not more than 0.5 .

Weight, strength, and opacity:

Weight: $(25$ by 40,500$)$
Weight: $(25$ by 38,500$)$

Folding endurance: A verage each direction, not less than

double folds.......................................... 75

Bursting strength: A verage not less than

Thickness: Average

$15 \mathrm{~mm}$ width-

Machine direction.

Across machine direction 4.0

Across machine direction

$\begin{array}{llll}40 & 50 & 60 & 70 \\ 38.0 & 47.5 & 57.0 & 66.5\end{array}$

$100 \quad 125 \quad 160 \quad 200$ $\begin{array}{lcccc} & 25 & 30 & 35 & 40 \\ 0.0035 & 0.0040 & 0.0050 & 0.0060 & 0.0060\end{array}$ $\begin{array}{lllll}4.0 & 5.0 & 6.0 & 7.0 & 8.0\end{array}$ $\begin{array}{cccc}2.5 & 3.0 & 3.5 & 4.0 \\ 86 & 89 & 90 & 90\end{array}$

Color, flnish, formation, cleanliness, and printing quality: Shall be in accordance with sample.

Testing methods: The testing methods shall be those approved by the Technical Association of the Pulp and Paper Industry. The physical specifications are for a relative humidity of 65 per cent and a tem急 perature of $70^{\circ} \mathrm{F}$., which are the testing conditions approved by this association.

\section{Class II. PAPERS OF HIGH PURITY FOR SEMIPERMANENT RECORDS}

Stock: Free from unbleached or ground wood fibers. Alpha-cellulose content not less than 80 per cent, Copper number not more than 2.0.

Acidity: pH value not less than 4.5 and acid number less than 25.

Rosin: Not more than 1.5 per cont.

Weight, strength, and opacity:

Weight: $(25$ by 40,500$)$

Folding endurance: Average each direction, not less than

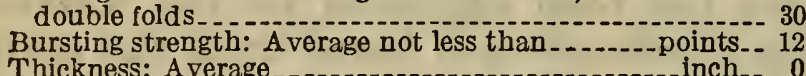

Thickness: Average

Tensile breaking strength: Average not less than kg per

$15 \mathrm{~mm}$ width-

Machine direction

Across machine direction

Opacity: Contrast ratio not less than

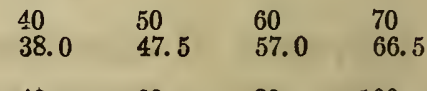

$40 \quad 60 \quad 80 \quad 100$.

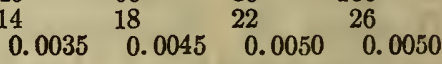

$3.0 \quad 4.0 \quad 5.0 \quad 6.0$

$\begin{array}{rrrr}1.5 & 2.0 & 2.5 & 3.0 \\ 86 & 89 & 90 & 90\end{array}$

Color, finish, formation, cleanliness, and printing quality: Shall be in accordance with sample.

Testing methods: The testing methods shall be those approved by the Technical Association of the Pulp and Paper Industry. The physical specifications are for a relative humidity of 65 per cent and a temperature of $70^{\circ} \mathrm{F}$., which are the testing conditions approved by this association.

\section{Class III. PAPERS OF FAIR PURITY FOR RECORDS REQUIRING MODERATE LONGEVITY}

Stock: Free from unbleached or ground wood fibers. Alpha-cellulose content not less than 70 per cent. Copper number not more than 3.5 .

Acidity: $\mathrm{pH}$ value not less than 4.5 and acid number less than 25.

Rosin: Not more than 2.0 per cent.

Weight, strength, and opacity:

Weight: $(25$ by 40,500$)$

-

Folding endurance: A verage each direction pounds.- 33. 3

not less than................................... 15

Bursting strength: Average not less than

Thickness: Average............. 0.0025

Tensile breaking strength: Average not less than $\mathrm{kg}$. per

$15 \mathrm{~mm}$. width -

Machine direction

3. 0

Across machine direction

Opacity: Contrast ratio not less than

Color, finish, formation, cleanliness, and printing quality: Shall be in accordance with sample.

Testing methods: 'The testing methods shall be those approved by the Technical Association of the Pulp

and Paper Industry. The physical specifications are for a relative humidity of 65 per cent and a tempera.

ture of $70^{\circ} \mathrm{F}$., which are the testing conditions approved by this association. 
TABLE 2.-Classification of book papers-Continued

Class IV. PAPERS FOR TEMPORARY USE

Stock, acidity, and rosin: No requirements;

Weight, strength, and opacity:

Weight: (25 by 40,500$)$.

Weight: $(25$ by 38,500$)$

Bursting strength: Average not less than _......... points.. 9

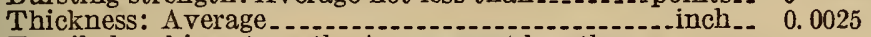

Tensile breaking strength: Average not less than

kg. per $15 \mathrm{~mm}$. width-

Machine direction.

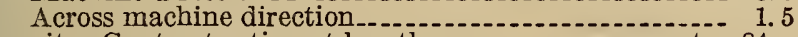

Opacity: Contrast ratio not less than printing quality: Shall be in accordance with sample.
Color, finish, formation, cleanliness, and

$\begin{array}{llll}40 & 50 & 60 & 70\end{array}$

$38.0 \quad 47.5 \quad 57.0 \quad 66.5$

$\begin{array}{llll}10 & 12 & 14 & 15\end{array}$

$\begin{array}{llll}0.0030 & 0.0033 & 0.0040 & 0.0050\end{array}$

$\begin{array}{rrrr}3.0 & 3.5 & 3.5 & 4.0 \\ 1.5 & 2.0 & 2.0 & 2.0 \\ 86 & 89 & 90 & 90\end{array}$

Testing methods: The testing methods shall be those approved by the Technical Association of the Pulp and Paper Industry. The physical specifications are for a relative humidity of 65 per cent and a temperature of $70^{\circ} \mathrm{F}$., which are the testing conditions approved by this association.

\section{SUMMARY}

1. In general, the book papers studied have a lower degree of purity and are affected to a greater extent by the heat test than writing papers made of the same kind and quality of fibrous materials.

2. The rosin content of most of the papers does not appear to be excessive, but the high acidity of many of them indicates an excessive use of alum, a practice which is very detrimental to the permanence of the papers.

3. The accelerated aging test shows that the change in purity, as measured by the change in alpha-cellulose content, is closely related to the loss of strength, as measured by the change in folding endurance, and that both of these factors are closely related to the acidity of the paper.

4. In general, the stability of these papers, as determined by the heat test, was dependent primarily on their chemical purity and had little relation to the source of the paper fiber or to its initial quality.

5 . The system of classification suggested distributes the papers among the various classes in about the ratio to be expected of commercial papers selected in the manner herein described.

\section{CONCLUSION}

The data produced in this study indicate that book papers, as they are commercially available at the present time, do not have, in general, as high permanence qualities as have been found in writing papers. This is probably largely true because in book papers printing quality appears, in general, to have been given more consideration than permanence, possibly because the purchaser of book papers is usually not so directly concerned with the preservation of printed records as is the purchaser of paper for written records. However, the fact that one of the papers may be classed as a paper of maximum permanence shows that it is possible to obtain permanence qualities in book papers comparable to those which can be obtained in writing papers, and at the same time preserve the printing qualities. A decided trend toward realization of this and of the need for more consideration of permanence qualities by book-paper makers and users is evident. The increasing cooperation in this respect between manufacturer, printer, and librarian will, no doubt, assist materially in making more certain that the paper in publications containing valuable records will have the desired longevity.

Washington, June 16, 1931. 